\title{
Molecular effects of lapatinib in patients with HER2 positive ductal carcinoma in situ
}

\author{
Laura G Estévez ${ }^{1 *}$, Ana Suarez-Gauthier ${ }^{1}$, Elena García², Cristina Miró ${ }^{1}$, Isabel Calvo ${ }^{1}$, María Fernández-Abad ${ }^{1}$, \\ Mercedes Herrero ${ }^{1}$, Manuel Marcos ${ }^{1}$, Cristina Márquez ${ }^{1}$, Fernando Lopez Ríos ${ }^{1}$, Sofía Perea ${ }^{1}$ and Manuel Hidalgo ${ }^{1,3}$
}

\begin{abstract}
Introduction: Human epidermal growth factor receptor 2 (HER2) amplification is frequent in ductal carcinoma in situ (DCIS) of the breast and is associated with poorly differentiated tumors and adverse prognosis features. This study aimed to determine the molecular effects of the HER2 inhibitor lapatinib in patients with HER2 positive DCIS.

Methods: Patients with HER2 positive DCIS received 1,500 mg daily of lapatinib for four consecutive weeks prior to surgical resection. Magnetic resonance imaging (MRI) was used to determine changes in tumor volume. The molecular effects of lapatinib on HER2 signaling (PI3K/AKT and RAS/MAPK pathways), cell proliferation (Ki67 and p27) and apoptosis (TUNEL) were determined in pre and post-lapatinib treatment samples.
\end{abstract}

Results: A total of 20 patients were included. Lapatinib was well tolerated with only minor and transient side effects. The agent effectively modulated HER2 signaling decreasing significantly pHER2 and pERK1 expression, together with a decrease in tumor size evaluated by MRI. There was no evidence of changes in Ki67.

Conclusions: Four weeks of neoadjuvant lapatinib in patients with HER2-positive DCIS resulted in inhibition of HER2 and RAS/MAPK signaling pathway.

Trial registration: 2008-004492-21 (Registered June 25th 2008).

\section{Introduction}

Data from the National Cancer Database estimate that 12 to $15 \%$ of newly diagnosed breast cancer in the US today is ductal carcinoma in situ (DCIS) [1]. DCIS is defined as a malignant proliferation of ductal cells of the breast that does not invade through the basal membrane. DCIS rarely presents as a palpable mass in the breast but is associated with microcalcifications seen in mammography. The broad implementation of screening programs has resulted in an increase from 5 to $25 \%$ in the number of patients diagnosed with this disease $[2,3]$.

Conventional management of DCIS includes surgical resection by either mastectomy or breast-conserving surgery and radiation therapy, depending on the extent of the disease [4,5]. Bilateral breast magnetic resonance imaging (MRI) is being used preoperatively with increasing frequency in women with DCIS, where it has shown high sensitivity for detecting high-grade lesions [6].

\footnotetext{
* Correspondence: lestevez@hmhospitales.com

'Breast Cancer Program, Centro Integral Oncológico Clara Campal, C/Oña 10, Madrid 28050, Spain

Full list of author information is available at the end of the article
}

Patients with hormone receptor-positive tumors benefit from adjuvant treatment with tamoxifen that decreases local and contralateral tumor failure [2-5]. Despite its excellent prognosis, in up to $10 \%$ of patients, DCIS will recur, often with invasive carcinoma [7].

One of the salient features of DCIS is the high expression of human epidermal growth factor receptor 2 (HER2). Although globally 20 to $30 \%$ of patients with invasive breast cancer express HER2, the expression is as high as 60 to $70 \%$ in patients with high-grade/comedo-type DCIS [8]. Expression of HER2 is associated with high proliferative grade, comedo-necrosis, and p53 mutation, and is inversely associated with the expression of hormone receptors $[9,10]$.

HER2 belongs to a receptor family that includes HER1, HER2, HER3, and HER4, respectively. These molecules are transmembrane tyrosine kinase receptors with partial homology that regulate cell growth and survival, as well as adhesion, migration, differentiation, and other cellular responses. HER2 are the preferred dimerization partners for the other HER family members [11]. When HER2 
induce dimerization with HER3, the phosphorylated (activated) tyrosine residues on the intracellular domain of HER2 activate the lipid kinase phosphoinositide 3-kinase (PI3K), which results in activation of the enzyme AKT transforming factor (AKT) promoting cell survival [11]. In contrast, when HER1 is the chosen partner for dimerization, the complex HER1-HER2 preferentially activate the rat sarcoma (RAS)/rapid accelerated fibrosarcoma (RAF)/mitogen-activated protein kinase (MAPK) cascades promoting cell proliferation [12]. HER2 is a validated therapeutic target in invasive breast cancer, leading to the hypothesis that inhibition of HER2 through anti-HER2 therapy could be beneficial for patients with DCIS.

Lapatinib, a reversible dual-kinase inhibitor of epidermal growth factor receptor (EGFR) and HER2, has activity in HER2-overexpressing breast cancer and is approved in combination with capecitabine for the treatment of patients with metastatic disease [13]. In early pharmacodynamic clinical studies, administration of lapatinib to patients with advanced breast cancer resulted in inhibition of HER2 signaling and induction of apoptosis [14-18]. At the recommended dose, the drug is well-tolerated with skin rash and diarrhea as the predominant toxicities. In rare cases, there were liver and cardiac adverse events [19].

The central hypothesis of the present study is that lapatinib inhibits HER2 signaling in DCIS patients resulting in cell proliferation arrest and apoptosis. The primary objective of the trial was to determine the pharmacodynamic effects of a 4-week course of lapatinib administered preoperatively to patients with HER2-positive DCIS measuring protein expression of phospho extracellularregulated kinase (pERK), phosphor-AKT (pAkt) and proliferative/apoptosis markers at baseline and post treatment. Secondary analysis included assessment of tumor response by MRI and the correlation with molecular biomarkers.

\section{Methods \\ Study design}

This study was a prospective, open-label, phase II trial, conducted at the Centro Integral Oncológico Clara Campal, Hospital Universitario Madrid, between December 2009 and June 2010. Patients were required to give written informed consent before inclusion in the study. The trial was conducted following the guidelines of the local Ethical Review Board and the Spanish Ministry of Health, and in accordance with Good Clinical Practices and the tenets of the Declaration of Helsinki. The protocol was approved by the Clinical Research Ethic Committee of Hospital Universitario Madrid Norte Sanchinarro (Madrid, Spain).

\section{Eligibility criteria}

Female patients with pathological diagnoses of HER2positive DCIS, according to American Society of Clinical
Oncology/College of American Pathologists (ASCO/CAP) guidelines [20], scheduled to undergo surgery by either lumpectomy or mastectomy were eligible. Other eligibility criteria included age $\geq 18$ years, Eastern Cooperative Oncology Group (ECOG) performance status 0 to 2; normal hematological (absolute neutrophil count $\geq 1.5$ cells $\times 10^{9} / \mathrm{L}$, platelets $\geq 75$ cells $\times 10^{9} / \mathrm{L}$, and hemoglobin $\geq 9 \mathrm{~g} / \mathrm{dl}$ ), liver (bilirrubin $\leq 1.25 \times$ upper limit of normal (ULN), aspartate transaminase (AST) and alanine transaminase $(\mathrm{ALT}) \leq 2.5 \times \mathrm{ULN}$ and alkaline phosphatase $\leq 2.5 \times \mathrm{ULN}$ ) and renal (creatinine $\leq 2.0 \mathrm{mg} / \mathrm{dL}$ or creatinine clearance $>40 \mathrm{ml} / \mathrm{min}$ ); and normal left ventricular ejection fraction. Lactating and pregnant women were excluded. Other exclusion criteria included previous treatment with lapatinib, contralateral breast mass, presence or suspicion of invasive carcinoma, axillary lymph nodes, malabsorption syndrome that could interfere with lapatinib exposure, any other serious cardiac, neurological or psychiatric disorder that in the investigator's opinion could compromise treatment safety and compliance, and concomitant use of CYP3A4 interacting agents. Women of fertile age were required to use appropriate contraception methods.

\section{Treatment administration}

Patients received lapatinib as single agent administered orally for 28 days (4 weeks) at a dose of 1,500 mg per day divided into six 250-mg capsules, following the package insert recommendations. Missed doses were not made up and no dose reductions were allowed. Treatment compliance was recorded in a patient diary. Patients who for whatever reason, received less than $80 \%$ of the prescribed dose were replaced.

\section{Study procedures and follow up}

Patients with histologically confirmed HER2-positive DCIS with measurable residual microcalcifications $(1 \mathrm{~cm})$ on mammography after initial diagnostic biopsy were counseled about the study. The first MRI of the breast was also performed after the diagnostic biopsy procedure. Written informed consent was obtained from all patients before study inclusion. Screening studies, including safety laboratory measurements and assessment of left ventricular ejection fraction (LVEF) were performed. Patients were visited on a weekly basis while on medication to monitor treatment compliance and toxicity. Physical examination, hematology and blood chemistry analysis were performed weekly. Treatment-related toxicities were recorded according to the National Cancer Institute (NCI) Common Toxicity Criteria (CTC) version 3 criteria and managed as per-package insert. The second breast MRI was performed on day 28 following the end of lapatinib treatment. Patients were schedule for surgery 3 to 4 weeks (range 1 to 4 ) after treatment completion. 


\section{MRI technique and interpretation}

Imaging studies (mammography and breast MRI) were both performed prior to biopsy procedure and definitive surgery, to permit an unbiased interpretation. Mammography and MRI scans were read and scored independently by one expert breast radiologist. All MRI examinations were performed on a commercially available 3-T system (MR Systems Achieva, Version 2.6.3.6; Philips Electronics 2010). Images were evaluated for areas of abnormal enhancement in the breast using previously described interpretation criteria [21]. Response assessment was defined according to response evaluation criteria in solid tumors (RECIST) [22].

\section{Tissue processing and biological studies}

Biological markers were measured before and after lapatinib treatment in order to evaluate treatment-induced changes. A fresh core needle-biopsy was obtained from each patient prior to lapatinib treatment. Tissue samples were immediately fixed in formalin after the extraction in the radiological room and embedded in paraffin (formalin-fixed paraffin-embedded, FFPE) for molecular tests. After lapatinib treatment, molecular tests were analyzed in representative tumor areas of the surgical specimen. In order to avoid suboptimal fixation of surgical specimens and resulting problem of loss of immunoreactivity of phosphoproteins in paraffin samples, surgical samples were processed as follows: tissue samples were moved from the operating room to the pathology room as soon as surgeons removed the sample from the patient. The samples were sliced at intervals of $5 \mathrm{~mm}$ and placed in large volume of formalin for 18 to 24 hours to allow the maximum penetration of formalin and to obtain the optimum fixation of samples. Median time from collection of samples was 8 minutes (range 1 (cores) to 15 (surgical specimens) minutes).

\section{Fluorescence in situ hybridization (FISH)}

To assess HER2 and EGFR amplification, fluorescence in situ hybridization (FISH) was performed in all samples using the PathVysion HER2 DNA Probe kit (Vysis) and the EGFR Probe kit (Vysis) with the DAKO Histology FISH Accessory Kit. HER2 gene amplification was considered when the ratio $H E R 2 / C E P 17$ was $\geq 2.2$. EGFR gene amplification was considered when the ratio EGFR/ CEP 7 was $\geq 2$.

\section{Immunohistochemistry (IHQ)}

Immunohistochemical studies were assessed in FFPE tissue for the following primary antibodies: HER2 (SP3 clone; 1:100 dilution; Spring); pHER2 (6B12 clone; 1/100 dilution; Cell Signaling Technology); EGFR (SPM341 clon; 1:25 dilution; Spring), phosphor-EGFR (pEGFR) (Tyr992) (Polyclonal; 1:25 dilution; Cell Signaling Technology),
AKT (11E7 clone; 1:50 dilution; Cell Signaling Technology); pAKT (Ser473)(736E11 clone; 1:50 dilution; Cell Signaling Technology), p44/42 MAPK (ERK 1) (137 F5 clone; 1:200 dilution; Cell Signaling Technology), pp44/42 MAPK (pERK1) (Thr202/Tyr204; E10) (20G11 clone; 1:200 dilution; Cell Signaling Technology), phosphatase and tensin homolog (PTEN) (28H6 clone; 1/100 dilution; Novocastra), p27 (SX53G8 clone; Ventana-Ready to use), and Ki67 (30 to 9 clone; Ventana-Ready to use). All antibodies except p27, Ki67 and HER2 were scored based on intensity from 0 to $3+$ and percentage of positive cells to generate a score $(H$-score) that ranges from 0 to 300 by multiplying these two parameters. p27 and Ki67 proliferative index were reported as percentage of positive nuclei in hot-spot areas. HER2 was scored following ASCO/CAP scoring criteria for immunohistochemistry. For biomarkers determined by $H$-score, overexpression was considered for scores $\geq 150$. The percentage of stained nuclei was evaluated independently of the intensity for Ki67 and p27 (cutoff $\geq 15 \%$ ).

\section{Mutational analysis}

Real-time polymerase chain reaction (PCR) mutational screening for phosphatidylinositol-4,5-bisphosphate 3kinase, catalytic subunit alpha (PIK3CA) (exons 9 and 20) was carried out using PCR and direct sequencing following standard protocols [23].

\section{Apoptosis cell analysis}

Terminal deoxynucleotidyl transferase-mediated deoxyuridine triphosphate-biotin nick-end labeling (TUNEL) was performed according to the manufacture instructions (Roche Diagnostics, Indianapolis, IN) [24]. The number of apoptotic cells was determined in each sample.

\section{Statistical and data analysis}

This was a single-arm phase-II study to test the hypothesis that lapatinib inhibits the HER2 pathway and induces apoptosis in patients with HER2-positive DCIS. The primary endpoint of the study was the pharmacodynamic effect of the drug on cell signaling, proliferation and apoptosis. The secondary endpoints included tumor changes on MRI. A sample size of 20 patients was arbitrarily selected for this study. For the analysis of the primary endpoint, each biomarker was evaluated before and after treatment and was summarized using descriptive statistics. A decrease or increase in the expression of each single biomarker was determined as a change in the $H$-score. The differences in this parameter before and after treatment were compared with the paired student $t$-test. The SPSS v.19 statistical program was used for all statistical analyses. A two-tailed value of $P<0.05$ was considered statistically significant. 


\section{Results}

Patient characteristics

A total of 20 patients, whose principal characteristics are listed in Table 1, were enrolled. Most patients had high-grade DCIS of non-comedo-type carcinoma and approximately half $(60 \%)$ of the subjects had positive hormone receptors.

\section{Treatment compliance, safety and type of surgery performed}

Seventeen patients completed the prescribed treatment. One patient received $250 \mathrm{mg}$ daily dose instead of the $1,500 \mathrm{mg}$ dose. In addition, two patients interrupted treatment by mistake (miss treatment on day 3 and on day 10) and unrelated side effect. Overall, the treatment was well-tolerated with no episode of grade-3 toxicity. The most frequent adverse events were skin reaction, with $35 \%$ of patients experiencing grade- 1 rash, and gastrointestinal toxicity, with $50 \%$ of patients experiencing grade- 1 to grade-2 diarrhea. The types of surgery performed were: conservative surgery $(n=13,65 \%)$ and mastectomy ( $\mathrm{n}=7,35 \%)$.

\section{Biomarkers before lapatinib treatment}

All the patients were positive for HER2 gene amplification with a median ratio of 7 (range 4 to 11.8). None of the patients had positive EGFR FISH, therefore, it was not evaluated on definitive surgery. At baseline, 16 patients (94\%) had pHER2 overexpression, 8 patients (42\%) had pAKT overexpression and 7 patients $(37 \%)$ had pERK1

Table 1 Patients' baseline characteristics

\begin{tabular}{ll}
\hline Characteristic & Value \\
\hline $\begin{array}{l}\text { Age, median (range) } \\
\text { perforn Cooperative Oncology Group }\end{array}$ & 51 (38 to 68) \\
0 & 20 \\
Histological subtype, number of patients & \\
Comedo carcinoma & 7 \\
Non-comedo carcinoma & 13 \\
Pathological subtype, number of patients & \\
Low grade & 0 \\
Intermediate grade & 4 \\
High grade & 16 \\
Without necrosis & 4 \\
Hormone receptor status, number of patients & \\
Estrogen receptor-positive & 12 \\
Estrogen receptor-negative & 7 \\
Progesterone receptor-positive & 9 \\
Progesterone receptor-negative & 10 \\
Unknown & 1 \\
\hline
\end{tabular}

cytoplasmatic overexpression. PTEN was not expressed in four patients (21\%). Sixteen patients (84\%) had Ki67 $\geq 15 \%$, whereas low p27-staining $(<15 \%)$ was observed in half of tumors. PI3KCA mutations were detected in only one patient $(6 \%)$.

\section{Effects on signaling pathways, cell proliferation, and apoptosis}

After 4 weeks of lapatinib treatment surgery was performed in the 20 patients. No evidence of carcinoma in situ was noted in two patients after definitive surgery. Biomarkers were evaluated in the 18 remaining patients. Tables 2 and 3 summarize the effects on signaling pathways in each patient individually and in the overall population, respectively. With regard to the HER2 signaling pathway, 13 patients had pre- and post-treatment samples for pHER2 assessment. Overall, there was a statistically significant decrement in pHER2 mean $H$-score from 237.08 pre-treatment to 79.85 post-treatment $(P<0.001)$. Of these 13 patients, 12 had pHER2 overexpression as defined in the study $(H$-score $\geq 150)$ and in 9 of them there was a reduction of $<150$ after treatment (Figure 1). Regarding the pERK1, 16 patients had paired samples before and after treatment. In the overall population with matched specimens there was a statistically significant decrease in the mean $H$-score from 117.44 pre-treatment to 45.19 post-treatment $(P<0.008)$. pERK1 expression decreased from a $H$-score $\geq 150$ to an $H$-score $<150$ in five our seven patients (Figure 2). Individually, three patients showed increased expression and in two patients no changes were detected in global matched specimens. Regarding pAKT, in 15 paired samples before and after treatment, no significant differences in the $H$-score $(P<0.620)$ were detected. In these matched samples, in four patients with pAKT overexpression at baseline, two had a decrease in the $H$-score below 150 after treatment. Finally, with regard to biomarker correlation, four out of nine patients (44\%) with pHER2 reduction $(H$-score $\geq 150$ to $H$-score $<150$ ), also presented a decrease in pERK1 expression with almost no changes in pAKT expression. Interestingly, one patient with marked inhibition of pHER2 and pERK1 paradoxically had a increase in pAKT.

Apoptosis index through a TUNEL assay was only performed in eight patients due to insufficient specimens and to three of them having an increase in apoptotic levels. The Ki67 index was analyzed before and after treatment in 16 patients with no significant differences in the global population. Likewise, the expression of p27 did not change significantly before and after lapatinib treatment (Table 3). Figure 3 depicts changes in the evaluated tumor biomarkers.

\section{Treatment response assessed by MRI}

All the patients included in this study underwent MRI before and after lapatinib treatment. MRI findings at the 
Table 2 Changes in $\boldsymbol{H}$-score in pHER2, pERK1 and pAKT

\begin{tabular}{|c|c|c|c|c|c|c|c|c|c|c|c|c|}
\hline \multirow[t]{3}{*}{ Patient number } & \multicolumn{6}{|c|}{ pHER2 expression } & \multicolumn{6}{|c|}{ pERK1 expression } \\
\hline & \multicolumn{3}{|c|}{ Pre-lapatinib } & \multicolumn{3}{|c|}{ Post-lapatinib } & \multicolumn{3}{|c|}{ Pre-lapatinib } & \multicolumn{3}{|c|}{ Post-lapatinib } \\
\hline & Intensity & Percentage & $H$-score & Intensity & Percentage & $H$-score & Intensity & Percentage & $H$-score & Intensity & Percentage & $H$-score \\
\hline 1 & N/A & $\mathrm{N} / \mathrm{A}$ & N/A & $2+$ & $90 \%$ & 180 & $3+$ & $26 \%$ & 78 & $1+$ & $46 \%$ & 46 \\
\hline 2 & $3+$ & $91 \%$ & 273 & $3+$ & $90 \%$ & 270 & $3+$ & $77+$ & 231 & $2+$ & $76 \%$ & 152 \\
\hline 3 & $3+$ & $90 \%$ & 270 & $2+$ & $43 \%$ & 86 & $2+$ & $80 \%$ & 160 & $1+$ & $2 \%$ & 2 \\
\hline 4 & $3+$ & $90 \%$ & 270 & $1+$ & $77 \%$ & 77 & $1+$ & $10 \%$ & 10 & $1+$ & $30 \%$ & 30 \\
\hline 5 & $3+$ & $90 \%$ & 270 & $\mathrm{~N} / \mathrm{A}$ & N/A & N/A & $1+$ & $10 \%$ & 10 & N/A & N/A & $\mathrm{N} / \mathrm{A}$ \\
\hline 6 & N/A & N/A & N/A & $3+$ & $90 \%$ & 270 & N/A & $\mathrm{N} / \mathrm{A}$ & N/A & $2+$ & $10 \%$ & 20 \\
\hline 7 & $2+$ & $47 \%$ & 94 & $1+$ & $5 \%$ & 5 & $1+$ & $20 \%$ & 20 & $1+$ & $8 \%$ & 8 \\
\hline 8 & $3+$ & $90 \%$ & 270 & $\mathrm{~N} / \mathrm{A}$ & N/A & $\mathrm{N} / \mathrm{A}$ & 0 & 0 & 0 & 0 & 0 & 0 \\
\hline 9 & $3+$ & $90 \%$ & 270 & 0 & 0 & 0 & $1+$ & $80 \%$ & 80 & 0 & 0 & 0 \\
\hline 10 & $3+$ & $50 \%$ & 150 & 0 & 0 & 0 & $1+$ & $90 \%$ & 90 & 0 & 0 & 0 \\
\hline 11 & $3+$ & $73 \%$ & 219 & N/A & N/A & $\mathrm{N} / \mathrm{A}$ & $1+$ & $10 \%$ & 10 & $\mathrm{~N} / \mathrm{A}$ & N/A & N/A \\
\hline 12 & N/A & $\mathrm{N} / \mathrm{A}$ & N/A & $1+$ & $5 \%$ & 5 & 0 & 0 & 0 & 0 & 0 & 0 \\
\hline 13 & $2+$ & $90 \%$ & 180 & $\mathrm{~N} / \mathrm{A}$ & N/A & $\mathrm{N} / \mathrm{A}$ & $1+$ & $10 \%$ & 10 & $\mathrm{~N} / \mathrm{A}$ & $\mathrm{N} / \mathrm{A}$ & $\mathrm{N} / \mathrm{A}$ \\
\hline 14 & $3+$ & $90 \%$ & 270 & $1+$ & $10 \%$ & 10 & $1+$ & $80 \%$ & 80 & $2+$ & $90 \%$ & 180 \\
\hline 15 & $3+$ & $90 \%$ & 270 & 0 & 0 & 0 & $2+$ & $95 \%$ & 190 & 0 & 0 & 0 \\
\hline 16 & $3+$ & $95 \%$ & 285 & $3+$ & $90 \%$ & 270 & $2+$ & $95 \%$ & 190 & 0 & 0 & 0 \\
\hline 17 & $3+$ & $50 \%$ & 150 & $2+$ & $50 \%$ & 100 & $1+$ & $95 \%$ & 190 & 0 & 0 & 0 \\
\hline 18 & $3+$ & $95 \%$ & 285 & 0 & 0 & 0 & $2+$ & $55 \%$ & 110 & 0 & 0 & 0 \\
\hline 19 & $3+$ & $75 \%$ & 225 & $1+$ & $10 \%$ & 10 & $3+$ & $70 \%$ & 210 & $2+$ & $10 \%$ & 20 \\
\hline 20 & $3+$ & 90 & 270 & $3+$ & $70 \%$ & 210 & $3+$ & $80 \%$ & 240 & $3+$ & $95 \%$ & 285 \\
\hline
\end{tabular}


Table 2 Changes in $\mathbf{H}$-score in pHER2, pERK1 and pAKT (Continued)

\begin{tabular}{|c|c|c|c|c|c|c|}
\hline \multirow[t]{3}{*}{ Patient number } & \multicolumn{6}{|c|}{ pAKt expression } \\
\hline & \multicolumn{3}{|c|}{ Pre-lapatinib } & \multicolumn{3}{|c|}{ Post-lapatinib } \\
\hline & Intensity & Percentage & $H$-score & Intensity & Percentage & $H$-score \\
\hline 1 & $1+$ & $5 \%$ & 5 & $1+$ & $40 \%$ & 40 \\
\hline 2 & $1+$ & $80 \%$ & 80 & $2+$ & $95 \%$ & 190 \\
\hline 3 & $2+$ & $50 \%$ & 100 & $3+$ & $3+$ & 285 \\
\hline 4 & $2+$ & $95 \%$ & 190 & $2+$ & $95 \%$ & 190 \\
\hline 5 & $3+$ & $90 \%$ & 270 & $\mathrm{~N} / \mathrm{A}$ & N/A & N/A \\
\hline 6 & N/A & N/A & N/A & $1+$ & $95 \%$ & 95 \\
\hline 7 & $2+$ & $90 \%$ & 180 & $1+$ & $95 \%$ & 95 \\
\hline 8 & $2+$ & $95 \%$ & 190 & $\mathrm{~N} / \mathrm{A}$ & N/A & N/A \\
\hline 9 & $1+$ & $90 \%$ & 90 & $1+$ & $90 \%$ & 90 \\
\hline 10 & $3+$ & $90 \%$ & 270 & $1+$ & $95 \%$ & 95 \\
\hline 11 & $3+$ & $90 \%$ & 270 & N/A & N/A & N/A \\
\hline 12 & 0 & 0 & 0 & 0 & 0 & 0 \\
\hline 13 & $3+$ & $90 \%$ & 270 & N/A & N/A & N/A \\
\hline 14 & 0 & 0 & 0 & $1+$ & $95 \%$ & 95 \\
\hline 15 & 0 & 0 & 0 & 0 & 0 & 0 \\
\hline 16 & $1+$ & $95 \%$ & 95 & $1+$ & $95 \%$ & 95 \\
\hline 17 & $1+$ & $95 \%$ & 95 & $1+$ & $95 \%$ & 95 \\
\hline 18 & $1+$ & $95 \%$ & 95 & $1+$ & $90 \%$ & 90 \\
\hline 19 & $1+$ & $95 \%$ & 95 & $1+$ & $95 \%$ & 95 \\
\hline 20 & $3+$ & $100 \%$ & 300 & $3+$ & $100 \%$ & 300 \\
\hline
\end{tabular}


Table 3 Biomarker analysis in matched specimens

\begin{tabular}{lllll}
\hline Biomarker & Number & Mean & SD & $P$-value \\
\hline Ki67 & 16 & 26.81 & 12.303 & 0.051 \\
Pre-lapatinib & 16 & 32.44 & 14.166 & \\
Post-lapatinib & & & & \\
p27 & 15 & 17.67 & 21.672 & 0.127 \\
Pre-lapatinib & 15 & 8.73 & 15.050 & \\
Post-lapatinib & & & & \\
pHER2 & 13 & 237.08 & 63.357 & 0.000 \\
Pre-lapatinib & 13 & 79.85 & 104.311 & \\
Post-lapatinib & & & & \\
pERK-1 & 16 & 117.44 & 84.806 & 0.005 \\
Pre-lapatinib & 16 & 45.19 & 84.673 & \\
Post-lapatinib & & & & \\
pAKt & 15 & 106.33 & 93.512 & 0.620 \\
Pre-lapatinib & 15 & 117.00 & 88.657 & \\
Post-lapatinib & 15 &
\end{tabular}

pHER2, phospho human epidermal growth factor receptor 2; pERK1, phosphor extracellular-regulated kinase 1. time of diagnosis showed Breast Imaging Reporting and Data System (BIRADS)-2 in eight patients with no evidence of abnormal images. These eight patients presented also with BIRADS-2 in the second MRI performed after lapatinib treatment. The remaining 12 patients had abnormal MRI before lapatinib treatment. The most common abnormality consisted of a non-nodular density with focal, linear, ductal, segmental or diffuse aspect. Of these 12 patients, 9 had a decrease in tumor volume after lapatinib treatment when both MRI studies were compared (Figure 4). In addition, in five out of nine patients (56\%) with a decrease in pHER2 expression, a reduction in tumor volume on MRI, or signal intensity, was also observed. The same situation was found in two patients $(50 \%)$ with reduced pERK1 where a decrease in the signal and tumor size on MRI was also observed (Table 4).

\section{Discussion}

This study is the first to report the use of neoadjuvant lapatinib for patients with HER2-positive DCIS. This pharmacodynamic study aimed to determine the molecular

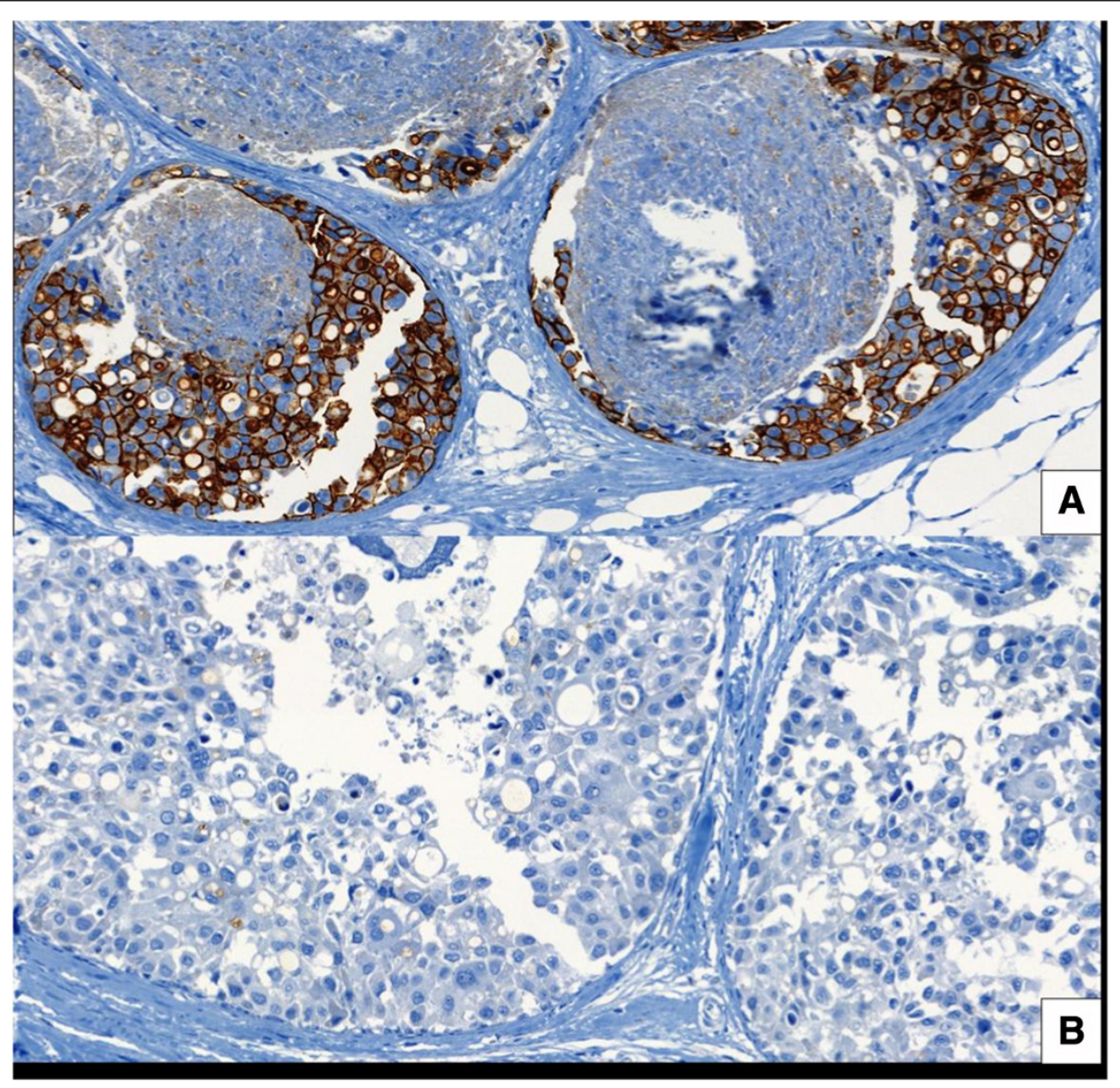

Figure 1 Phospho human epidermal growth factor receptor 2 (pHER2) protein expression. (A) Positive expression before lapatinib treatment. (B) Negative expression after lapatinib treatment. 


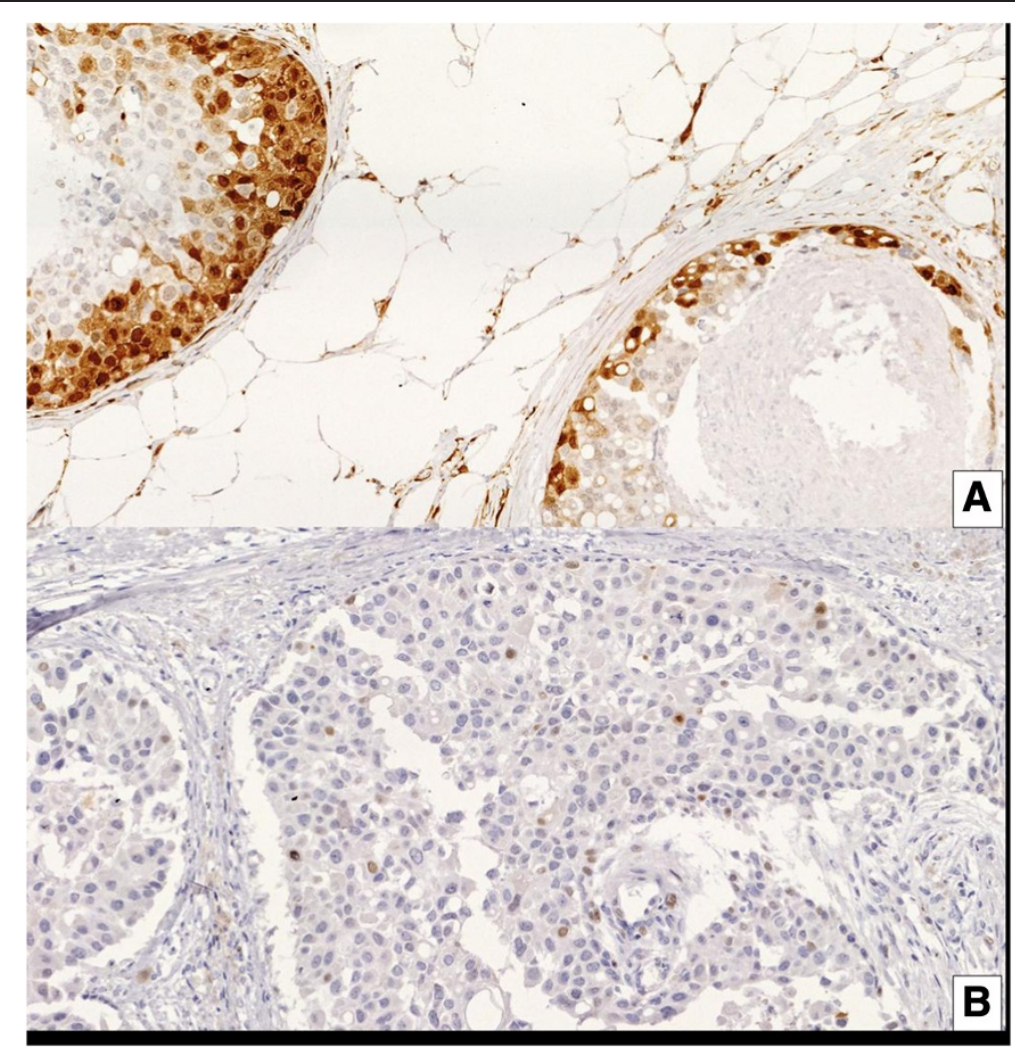

Figure 2 Phosphor extracellular-regulated kinase 1 ( $p E R K$ ) protein expression. (A) Positive expression before lapatinib treatment. (B) Negative expression after lapatinib treatment.

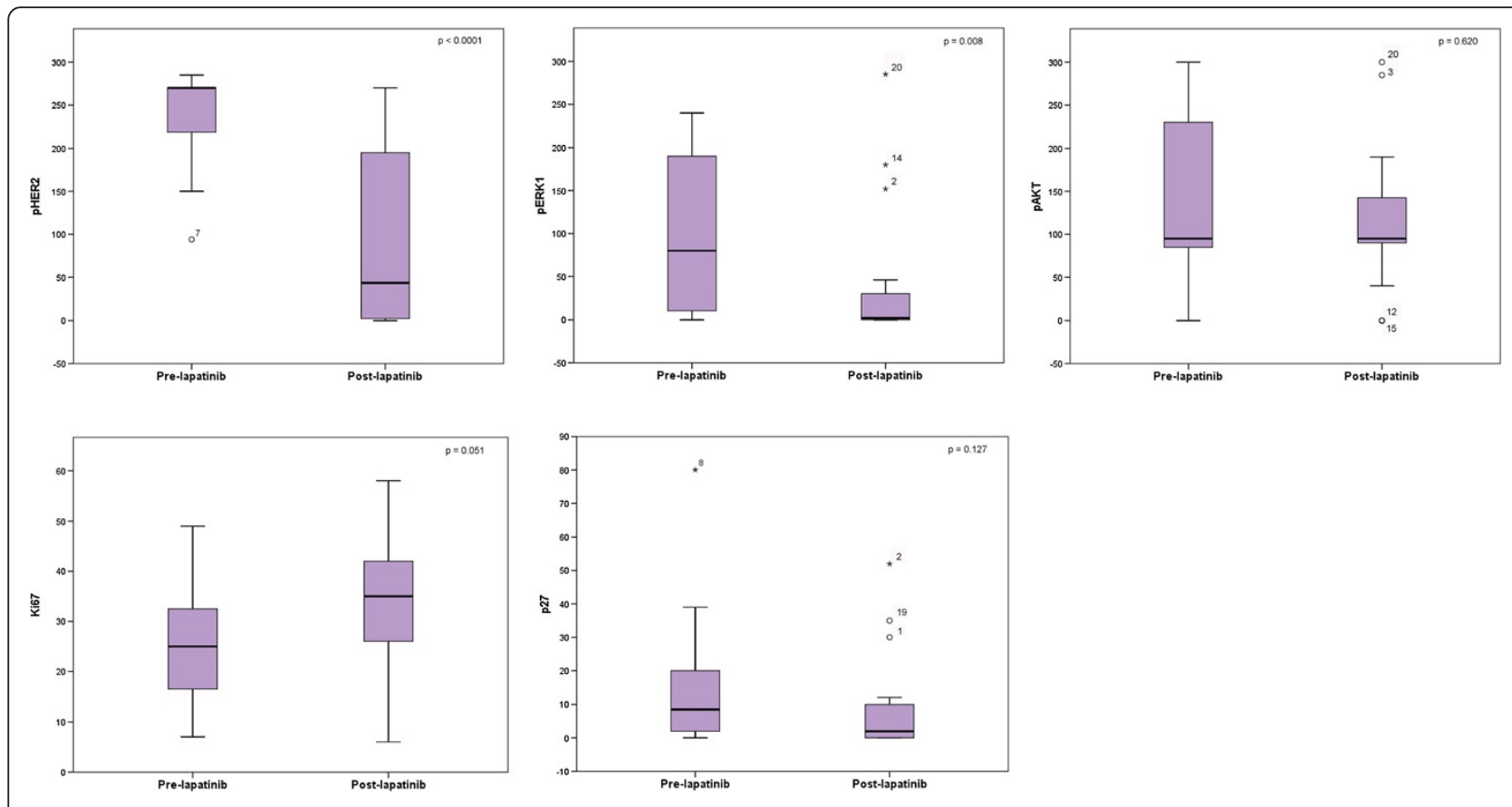

Figure 3 Changes in biomarkers before and after lapatinib. pHER2, phospho human epidermal growth factor receptor 2; pERK1, phosphor extracellular-regulated kinase 1. 

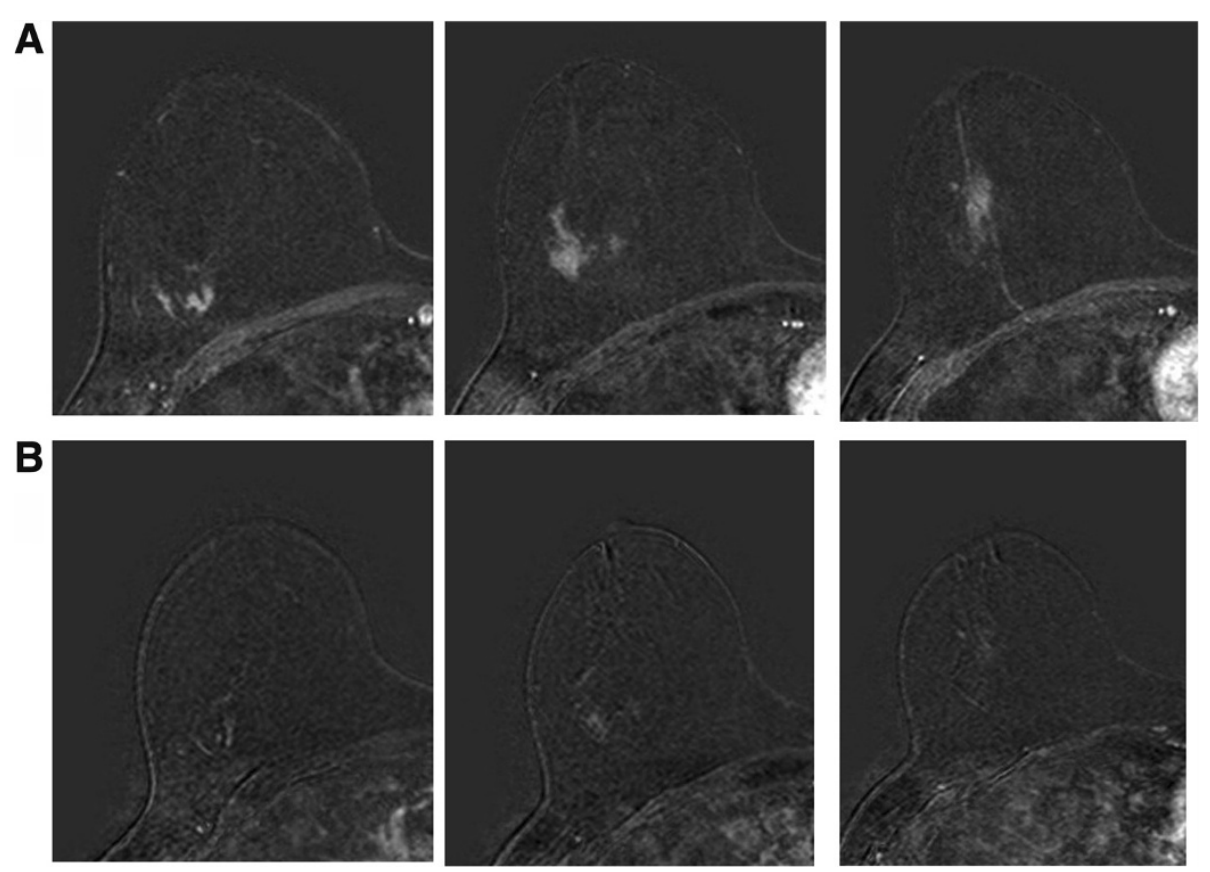

Figure 4 Treatment response assessed by MRI before (A) and after (B) lapatinib treatment.

activity of the HER2 inhibitor lapatinib in patients with HER2-positive DCIS. Using a preoperative window of treatment administration for 4 weeks prior to surgical resection, the study showed that the agent is effective in downregulating the HER2 pathway, with slight tendency in the $R A S / M A P K$ pathways with virtually no effect against the $P I 3 K / A K T$ pathway. In addition, the agent induced apoptosis as previously seen in invasive breast cancer; however the number of patients was too small to draw any strong conclusions. These molecular effects were associated with significant tumor regression on MRI.

The antitumor effects of HER2 inhibitors require the modulation of key signaling pathways and cell cycle/apoptosis regulatory molecules that mediate the transforming effects of HER2 activation. Recent data in invasive breast cancer support the observation that activation of the PI3K pathway or loss of phosphatase and tensin homolog (PTEN) is associated with resistance to trastuzumab $[25,26]$. Mechanisms for lapatinib resistance are less well-established. In a recent trial that aimed to evaluate the differential effects of trastuzumab and lapatinib under low and normal PTEN conditions in HER2overexpressing breast cancer cell lines, lapatinib was effective and decreased both pMAPK and pAKT under low PTEN conditions. In addition, lapatinib showed a significant decrease in Ki67 [27]. As opposed to these findings, the results in the present study showed an increase in Ki67 after lapatinib treatment. It is difficult to explain such results based on well-established scientific evidence reported in the literature as well as on the mechanism of action of lapatinib. Therefore, it is imperative to confirm such results in other patient cohorts that receive the same treatment scheme.

A similar trial with trastuzumab in HER2-positive DCIS was conducted at the MD Anderson Cancer Center in Houston by Kuerer et al. [28]. In that study, a single loading dose of $8 \mathrm{mg} / \mathrm{kg}$ of trastuzumab was administered to test the biological effects of the agent on DCIS 3 to 4 weeks before surgical resection. In that study, 12 patients received the study drug and 12 patients served as controls with no treatment. Pre- and post-treatment tissues with DCIS were studied for proliferation and apoptosis in patients treated with trastuzumab. The conclusion was that single-dose monotherapy with trastuzumab for patients with HER2-positive DCIS does not result in significant clinical, histologic, proliferative, or apoptotic changes, but results in antibodydependent cell-mediated cytotoxicity (ADCC)-mediated response through natural killer (NK) cells, and may also induce humoral immunity in a T-cell-dependent manner.

One of the weaknesses of the present study is the lack of a control group. The fact that the present study was performed in only one institution, and not being a multicenter study, prevented the recruitment of a control arm of DCIS untreated patients.

The prognosis of patients with DCIS with conventional surgical and medical treatment is very good and results 


\begin{tabular}{|c|c|c|c|c|c|}
\hline $\begin{array}{l}\text { Patient } \\
\text { number }\end{array}$ & $\begin{array}{l}\text { MRI } \\
\text { response }\end{array}$ & $\begin{array}{l}\text { pHER2 } \\
\text { reduction }\end{array}$ & $\begin{array}{l}\text { pERK1 } \\
\text { reduction }\end{array}$ & $\begin{array}{l}\text { MRI-pHER2 } \\
\text { correlation }\end{array}$ & $\begin{array}{l}\text { MRI-pERK1 } \\
\text { correlation }\end{array}$ \\
\hline 1 & $\begin{array}{l}\text { No } \\
\text { Change* }\end{array}$ & NA & No & NA & NA \\
\hline 2 & $P R$ & No & No & No & No \\
\hline 3 & PR & Yes & Yes & Yes & Yes \\
\hline 4 & PR & Yes & No & Yes & No \\
\hline 5 & $\begin{array}{l}\text { No } \\
\text { change* }\end{array}$ & NA & NA & NA & NA \\
\hline 6 & $\begin{array}{l}\text { No } \\
\text { change* }\end{array}$ & NA & NA & NA & NA \\
\hline 7 & $\begin{array}{l}\text { No } \\
\text { change* }\end{array}$ & No & No & NA & NA \\
\hline 8 & PR & No & No & No & No \\
\hline 9 & PR & Yes & No & Yes & No \\
\hline 10 & PR & Yes & No & Yes & No \\
\hline 11 & PR & NA & NA & No & No \\
\hline 12 & $\begin{array}{l}\text { No } \\
\text { change* }\end{array}$ & NA & No & NA & NA \\
\hline 13 & $\begin{array}{l}\text { No } \\
\text { change* }\end{array}$ & NA & NA & NA & NA \\
\hline 14 & SD & Yes & No & No & No \\
\hline 15 & $\begin{array}{l}\text { No } \\
\text { change* }\end{array}$ & Yes & Yes & NA & NA \\
\hline 16 & SD & No & No & No & No \\
\hline 17 & $P R$ & Yes & Yes & Yes & Yes \\
\hline 18 & PR & Yes & No & Yes & No \\
\hline 19 & $\begin{array}{l}\text { No } \\
\text { change* }\end{array}$ & Yes & Yes & NA & NA \\
\hline 20 & SD & No & No & No & No \\
\hline
\end{tabular}

pHER2, phospho human epidermal growth factor receptor 2; pERK1, phosphor extracellular-regulated kinase 1; SD, stable disease (response evaluation criteria in solid tumors (RECIST) criteria); NA, not applicable; PR, partial response (RECIST criteria); no change*, patients with Breast Imaging Reporting and Data System (BIRADS)-2 at diagnosis.

in cure in the majority of patients. Some patients with extensive disease and aggressive tumors, however, need a mastectomy for full tumor eradication. Interestingly, DCIS has HER2 amplification with higher frequency than invasive tumors and this HER2-positive DCIS is often associated with comedo DCIS which carries twice the risk of local recurrence as compared to non-comedo DCIS $[29,30]$. Because of the availability of HER2 inhibitors for cancer treatment, testing whether or not these agents are effective in HER2 DCIS is reasonable. However, given the excellent prognosis of this disease, determining their clinical effect will require a large and lengthy study. In this sense, the ongoing National Surgical Adjuvant Breast Project (NSABP) B-43 phase-III randomized trial in patients with HER2-overexpressing DCIS tests the efficacy of adding trastuzumab to conventional surgical and radiation therapy treatment.

The present study used a preoperative 4-week window between diagnosis and definitive surgery to assess biomarker modulation. Data from the literature have shown that tumor manipulation due to diagnostic procedures such as core biopsies do not induce either molecular changes or changes in biomarker determination in the post-treatment biopsy [31]. However, loss of immunoreactivity of phosphorylated antibodies in paraffin samples may produce significant differences in the results associated with the inappropriate elapsing time of sample fixation. In fact, extreme loss of immunoreactive p-Akt and p-Erk1/2 during routine fixation of primary breast cancer has been reported [32]. However, the present study utilized optimum elapsing time for sample fixation in order to overcome this problem.

In vitro studies have shown that blockade of EGFR and HER2 receptors by monoclonal antibodies inhibits cell proliferation. In our study, lapatinib affected HER2 signaling pathways and downregulated the RAS/MAPK cascade throughout moderate reduction in pERK1 protein expression, with no effect in pAKT expression. These biological effects were reflected in signal reduction on MRI. A paradoxical activation of pAKT was observed in one patient with inactivation of pHER2 and pERK1. This negative feedback-loop has been recently described as resulting from HER3 hyperactivation, suggesting that dual inhibition of both pathways should be the most adequate and effective therapy [33].

Several important issues, including the optimal biomarker, methods to determine such biomarkers, and scores to discriminate between positive and negative biomarkers, remain unknown. Unlike for HER2 testing, for which there is currently an international consensus for positive or negative status, there is no consensus on other biomarkers in the HER2 signaling pathway that are indicative of pathway inhibition [34]. The present study evaluates the downregulation of biomarkers in the RAS/ $M A P K$ and PI3K/AKT pathways by IHC before and after lapatinib administration in order to determine the mechanism of action of this drug in DCIS. The result of this study is that lapatinib results in inhibition of $H E R$ and RAS/MAPK pathways with no effect in PI3K/AKT as well as the Ki67 proliferative index. However, there may be other elements in the HER signaling pathway that are more relevant to the actions of lapatinib. In addition, we have arbitrarily selected an $H$-score cutoff point to determine positive and negative expression, which may not necessarily be biologically relevant. A study correlating biomarker modulation with pathological response may be more informative in that regard. The fact that over half of the patients with pHER2 downregulation had a reduction in tumor size measured by MRI might indicate a possible activity of lapatinib in DCIS. Indeed, two 
patients had no residual tumor at the time of surgery in this study. While we cannot rule out that these small tumors may have been resected with the diagnostic biopsy, it suggests that lapatinib is effective in this setting. Therefore, other methods to assess biological activity such as gene expression profile, albeit more expensive and time-consuming, may be more informative. Finally, the molecular events leading to significant tumor regression on MRI were essentially not captured by this study. This may require a larger study and the use of more sensitive and comprehensive molecular tests.

The fundamental question now, having documented drug safety and molecular effects, is what to do next. Certainly, a randomized clinical trial comparing lapatinib versus no treatment in HER2-amplified poor-prognosis DCIS with local failure, as the primary endpoint would be required before this treatment can be recommended. Because of the excellent prognosis, the study would include a large number of patients, with a long follow up. Because lapatinib is associated with tumor regression in this setting, a trial assessing whether preoperative administration of the agent results in tumor down-staging, increasing the proportion of patients who can have a breast-sparing procedure, would also be interesting.

To our knowledge, this is the first study evaluating the role of neoadjuvant lapatinib in DCIS. Despite the short half-life of lapatinib (24 hours), its molecular effect in tumoral cells was observed beyond the expected time (4-week period between the last dose of lapatinib and the time of surgery). This phenomenon would indicate that the molecular changes induced by lapatinib are maintained over time. However, this hypothesis should be confirmed with further research.

\section{Conclusions}

The present study shows the feasibility of a short-window treatment with a targeted agent in patients with DCIS. The results demonstrate that standard-dose lapatinib results in signaling modulation by decreasing the RAS/ $M A P K$ signaling pathway in patients with HER2-positive DCIS. Additional studies are needed to determine if this strategy results in improved patient outcome.

\footnotetext{
Abbreviations

ADCC: antibody-dependent cell mediated cytoxicity; AKT: AKT transforming factor; ALT: alanine transaminase; ASCO/CAP: American Society of Clinical Oncology/College of American Pathologists; AST: aspartate transaminase; BIRADS-2: Breast Imaging Reporting and Data System; CTC: common toxicity criteria; DCIS: ductal in situ carcinoma; ECOG: Eastern Cooperative Oncology Group; EGFR: epidermal growth factor receptor; ERK: extracellular-regulated kinase; FFPE: formalin-fixed paraffin-embedded; FISH: fluorescence in situ hybridization; HER2: human epidermal growth factor receptor 2; LVEF: left ventricular ejection fraction; MAPK: mitogen-activated protein kinase; MRI: magnetic resonance imaging; NCl: National Cancer Institute; NSABP: National Surgical Adjuvant Breast Project; pAKT: phosphor AKT; PCR: polymerase chain reaction; pEGFR: phospho EGFR; pERK: phospho ERK; PI3K: phosphatidylinositol-4,5-biphosphate 3-kinase;

PI3KCA: phosphatidylinositol-4,5-biphosphate 3-kinase, catalytic subunit
}

alpha; PTEN: phosphatase and tensin homolog; RAF: rapid accelerated fibrosarcoma; RAS: rat sarcoma; RECIST: response evaluation criteria in solid tumors; TUNEL: terminal deoxynucleotide transferase-mediated deoxyuridine triphosphate-biotin nick-end labelling; UNL: upper normal limit.

\section{Competing interests}

The authors declare that they have no competing interests.

\section{Authors' contributions}

LGE: study conception, study design, coordination, patient enrollment, statistical analysis and manuscript preparation. AS: molecular and pathological studies. EG: molecular and pathological studies. CM: radiological studies. IC: patient enrollment, study design and manuscript preparation. MFA: patient enrollment, study design and manuscript preparation. $\mathrm{MH}$ : surgical procedures. MM: surgical procedures. CM: radiological studies. FLR: molecular and pathological studies. SP: coordination and manuscript preparation. $\mathrm{MH}$ : statistical analysis, coordination, and manuscript preparation. All authors have read and approved the final version of the manuscript.

\section{Author details}

${ }^{1}$ Breast Cancer Program, Centro Integral Oncológico Clara Campal, C/Oña 10, Madrid 28050, Spain. ²Pathology Department, Hospital Universitario Fundación Alcorcón, Madrid 28922, Spain. ${ }^{3}$ Clinical Research Program, Spanish National Cancer Research Center, Madrid 28029, Spain.

Received: 20 May 2013 Accepted: 1 July 2014

Published: 4 September 2014

\section{References}

1. Winchester DJ, Menk HR, Winchester DP: National treatment trends for ductal carcinoma in situ of the breast. Arch Surg 1997, 132:660-665.

2. Ben-David MA, Sturtz DE, Griffith KA, Douglas KR, Hayman JA, Lichter AS, Pierce $L J$ : Long-term results of conservative surgery and radiotherapy for ductal carcinoma in situ using lung density correction: the University of Michigan experience. Breast J 2007, 13:392-400.

3. Hieken TJ, Cheregi J, Farolan M, Kim J, Velasco JM: Predicting relapse in ductal carcinoma in situ patients: an analysis of biologic markers with long-term follow-up. Am J Surg 2007, 194:504-506.

4. Patani N, Cutuli B, Mokbel K: Current management of DCIS: a review. Breast Cancer Res Treat 2008, 111:1-10.

5. Vazquez B, Rousseau D, Hurd TC: Surgical management of breast cancer. Semin Oncol 2007, 34:234-240.

6. Kuhl CK, Schrading S, Bieling HB, Wardelmann E, Leutner CC, Koenig R, Khun W, Schild HH: MRI for diagnosis of pure ductal carcinoma in situ: a prospective observational study. Lancet 2007, 370:485-492.

7. Estévez LG, Alvarez I, Seguí MA, Muñoz M, Margelí M, Miró C, Rubio C, Lluch A, Tusquets I: Current perspectives of ductal carcinoma in situ. Cancer Treat Rev 2010, 36:507-517.

8. Hoque A, Sneige N, Sahin AA: Her-2/neu gene amplification in ductal carcinoma in situ of the breast. Cancer Epidemiol Biomarkers Prev 2002, 11:587-590

9. Lindemann K, Resau J, Nahrig J, Kort E, Leeser B, Annecke K, Welk A, Schäefer J, Vande Woude GF, Lengyel E, Harbeck: Differential expression of c-Met, its ligand HGF/SF and HER2/neu in DCIS and adjacent normal breast tissue. Histopathology 2007, 51:54-62.

10. Steinman S, Wang J, Bourne P, Yang Q, Tang P: Expression of cytokeratin markers, ER-alpha, PR, HER-2/neu, and EGFR in pure ductal carcinoma in situ (DCIS) and DCIS with co-existing invasive ductal carcinoma (IDC) of the breast. Ann Clin Lab Sci 2007, 37:127-134.

11. Howe LR, Brown PH: Targeting the HER/EGFR/ErbB family to prevent breast cancer. Cancer Prev Res (Phila) 2011, 4:1149-1157.

12. Dillon RL, White DE, Muller WJ: The phosphatidyl inositol 3-kinase signaling network: implications for human breast cancer. Oncogene 2007, 26:1338-1345.

13. Tevaarwerk AJ, Kolesar JM: Lapatinib: a small-molecule inhibitor of epidermal growth factor receptor and human epidermal growth factor receptor-2 tyrosine kinases used in the treatment of breast cancer. Clin Ther 2009, 31:2332-2348.

14. Giampaglia M, Chiuri VE, Tinelli A, De Laurentiis M, Silvestris N, Lorusso V: Lapatinib in breast cancer: clinical experiences and future perspectives. Cancer Treat Rev 2010, 36:S72-S79. 
15. Moy B, Kirkpatrick P, Kar S, Goss P: Lapatinib. Nat Rev Drug Discov 2007, 6:431-432.

16. Geyer CE, Forster J, Lindquist D, Chan S, Romieu G, Pienkowski T, Jagiello-Gruszfeld A, Crown J, Chan A, Kaufman B, Skarlos D, Campone M, Davidson N, Berger M, Oliva C, Rubin SD, Stein S, Cameron D: Lapatinib plus capecitabine for HER2-positive advanced breast cancer. $N$ Engl J Med 2006, 355:2733-2743.

17. Konecny GE, Pegram MD, Venkatesan N, Finn R, Yand G, Rahmeh M, Untch M, Rusnak DW, Spehar G, Mullin RJ, Keith BR, Gilmer TM, Berger M, Podratz KC, Slamon DJ: Activity of the dual kinase inhibitor lapatinib (GW572016) against HER-2-overexpressing and trastuzumab-treated breast cancer cells. Cancer Res 2006, 66:1630-1639.

18. Xia W, Bisi J, Strum J, Liu L, Carrick K, Graham KM, Treece AL, Hardwicke MA, Dush M, Liao Q, Westlund RE, Zhao S, Bacus S, Spector NL: Regulation of survivin by ErbB2 signaling: therapeutic implications for ErbB2overexpressing breast cancers. Cancer Res 2006, 66:1640-1647.

19. Moy B, Goss PE: Lapatinib-associated toxicity and practical management recommendations. Oncologist 2007, 12:756-765.

20. Wolff AC, Hammond ME, Schwartz JN, Wolff AC, Hammond ME, Schwartz JN, Hagerty KL, Allred DC, Cote RJ, Dwosett M, Fitzgibbons PL, Hann WM, Langer A, Mc Shane LM, Paik S, Pegram MD, Perez EA, Press MF, Rhodes A, Sturgeon C, Taube SE, Tubbs R, Vance GH, van de Vijver M, Wheeler TM, Hayes DF, American Society of Clinical Oncology; College of American of Pathologists: American Society of Clinical Oncology/College of American Pathologists guideline recommendations for human epidermal growth factor receptor 2 testing in breast cancer. J Clin Oncol 2007, 25:118-145.

21. Morakkabati-Spitz N, Leutner C, Schild HH, Träber F, Kuhl CK: Diagnostic usefulness of segmental and linear enhancement in dynamic breast MRI. Eur Radiol 2005, 15:2010-2017.

22. Nishino M, Jackman DM, Hatabu H, Yeap BY, Cioffredi LA, Yap JT, Janne PA Johnson BE, Van den Abbeele AD: New Response Evaluation Criteria in Solid Tumors (RECIST) guidelines for advanced non-small cell lung cancer: comparison with original RECIST and impact on assessment of tumor response to targeted therapy. AJR Am J Roentgenol 2010, 195:W221-W228.

23. Angulo B, Suarez-Gauthier A, Lopez-Rios F, Medina PP, Conde E, Tang M, Soler G, Lopez-Encuentra A, Cigudosa JC, Sanchez-Cespedes M: Expression signatures in lung cancer reveal a profile for EGFR-mutant tumours and identify selective PIK3CA overexpression by gene amplification. J Pathol 2008, 214:347-356.

24. Loo DT: In situ detection of apoptosis by the TUNEL assay: an overview of techniques. Methods Mol Biol 2011, 682:3-13.

25. Rexer BN, Arteaga CL: Intrinsic and acquired resistance to HER2-targeted therapies in HER2 gene-amplified breast cancer: mechanisms and clinical implications. Crit Rev Oncog 2012, 17:1-16.

26. Berns K, Horlings HM, Hennessy BT, Madiredjo M, Hijmans E, Veelen K, Linn SC, Gonzalez-Angulo AM, Stemke-Hale K, Hauptmann M, Beijersbergen RL, Mills $G B$, van de Vijver MJ, Bernards R: A functional genetic approach identifies the PI3K pathway as a major determinant of trastuzumab resistance in breast cancer. Cancer Cell 2007, 12:395-402.

27. Dave B, Migliaccio I, Gutierrez MC, Wu MF, Chamness GC, Wong H, Narasanna A, Chakrabarty A, Hilsenbeck SG, Huang J, Rimawi M, Schiff R, Arteaga C, Osborne CK, Chang JC: Loss of phosphatase and tensin homolog or phosphoinositol-3 kinase activation and response to Trastuzumab or Lapatinib in human epidermal growth factor receptor 2-overexpressing locally advanced breast cancers. J Clin Oncol 2011, 29:166-173.

28. Kuerer HM, Buzdar AU, Mittendorf EA, Esteva FJ, Lucci A, Vence LM, Radvanyi K, Meric-Bernstam F, Hunt KK, Symmans WF: Biologic and immunologic effects of preoperative trastuzumab for ductal carcinoma in situ of the breast. Cancer 2011, 117:39-47.

29. Boughey JC, González RJ, Bonner E, Kuerer HM: Current treatment and clinical trial developments for ductal carcinoma in situ of the breast. Oncologist 2007, 12:1276-1287.

30. Boyages J, Delaney G, Taylor R: Predictors of local recurrence after treatment of ductal carcinoma in situ: A meta-analysis. Cancer 1999, 85:616-628

31. Mohsin SK, Weiss HL, Gutierrez MC, Chamness GD, Schiff R, Digiovanna MP, Wand CX, Hilsenbeck SG, Osborne CK, Allred CD, Elledge R, Chang JC: Neoadjuvant trastuzumab induces apoptosis in primary breast cancers. J Clin Oncol 2005, 23:2460-2468.
32. Pinhel IF, Macneill FA, Hills MJ, Salter J, Detre $S, A^{\prime}$ Hern $R$, Nerurkar $A$, Osin $P$, Smith IE, Dowsett M: Extreme loss of immunoreactive p-Akt and p-Erk1/2 during routine fixation of primary breast cancer. Breast Cancer Res 2010, 12:R76.

33. Garrett JT, Olivares MG, Rinehart C, Granja-Ingram ND, Sánchez V, Chakrabarty A, Dave B, Cook RS, Pao W, McKinely E, Manning HC, Chang J, Arteaga CL: Transcriptional and posttranslational up-regulation of HER3 (ErbB3) compensates for inhibition of the HER2 tyrosine kinase. Proc Natl Acad Sci USA 2011, 108:5021-5026.

34. Bose S, Chandran S, Mirocha JM, Bose N: The Akt pathway in human breast cancer: a tissue-array-based analysis. Mod Pathol 2006, 19:238-245.

doi:10.1186/bcr3695

Cite this article as: Estévez et al:: Molecular effects of lapatinib in patients with HER2 positive ductal carcinoma in situ. Breast Cancer Research 2014 16:R76.

\section{Submit your next manuscript to BioMed Central and take full advantage of:}

- Convenient online submission

- Thorough peer review

- No space constraints or color figure charges

- Immediate publication on acceptance

- Inclusion in PubMed, CAS, Scopus and Google Scholar

- Research which is freely available for redistribution 